\title{
Video Article \\ Using Robotic Systems to Process and Embed Colonic Murine Samples for Histological Analyses
}

\author{
Fulvia Milena Cribiù ${ }^{1}$, Claudia Burrello ${ }^{{ }^{2}}$, Roberta Tacchi ${ }^{1}$, Francesca Boggio ${ }^{1}$, Dario Ricca ${ }^{1}$, Flavio Caprioli ${ }^{3,4}$, Stefano Ferrero ${ }^{1}$, \\ Federica Facciotti ${ }^{2}$ \\ ${ }^{1}$ Pathology Unit, Fondazione IRCCS Cà Granda, Ospedale Maggiore Policlinico \\ ${ }^{2}$ Department of Experimental Oncology, European Institute of Oncology \\ ${ }^{3}$ Gastroenterology and Endoscopy Unit, Fondazione IRCCS Cà Granda, Ospedale Maggiore Policlinico \\ ${ }^{4}$ Department of Pathophysiology and Transplantation, Università degli Studi di Milano \\ *These authors contributed equally
}

Correspondence to: Federica Facciotti at Federica.Facciotti@ieo.it

URL: https://www.jove.com/video/58654

DOI: doi:10.3791/58654

Keywords: Immunology and Infection, Issue 143, histopathological analysis, murine models, automation, embedding system, standardization, murine intestinal specimens

Date Published: 1/7/2019

Citation: Cribiù, F.M., Burrello, C., Tacchi, R., Boggio, F., Ricca, D., Caprioli, F., Ferrero, S., Facciotti, F. Using Robotic Systems to Process and Embed Colonic Murine Samples for Histological Analyses. J. Vis. Exp. (143), e58654, doi:10.3791/58654 (2019).

\section{Abstract}

The understanding of human diseases has been greatly expanded thanks to the study of animal models. Nonetheless, histopathological evaluation of experimental models needs to be as rigorous as that applied for human samples. Indeed, drawing reliable and accurate conclusions is critically influenced by the quality of tissue section preparation. Here, we describe a protocol for histopathological analysis of murine tissues that implements several automated steps during the procedure, from the initial preparation to the paraffin embedding of the murine samples. The reduction of methodological variables through rigorous protocol standardization from automated procedures contributes to increased overall reliability of murine pathological analysis. Specifically, this protocol describes the utilization of automated processing and embedding robotic systems, routinely used for the tissue processing and paraffin embedding of human samples, to process murine specimens of intestinal inflammation. We conclude that the reliability of histopathological examination of murine tissues is significantly increased upon introduction of standardized and automated techniques.

\section{Video Link}

The video component of this article can be found at https://www.jove.com/video/58654/

\section{Introduction}

In the last decades, several experimental models have been developed to dissect the pathogenic mechanisms leading to human diseases ${ }^{1,2}$. In order to assess the severity of a disease, researchers must evaluate the effect of a treatment and study the cytological and histological architectural variations or the amount of inflammation ${ }^{3}$. To perform on those experimental models, detailed histopathological analyses are needed, often comparing murine and human samples ${ }^{4,5}$.

Additionally, human samples are commonly processed and scored by histopathology core facilities and experienced human pathologists through standardized histopathological criteria and methods. Conversely, murine tissues are usually fixed, embedded and analyzed by researchers with limited experience of histopathological protocols. The quality and reliability of histopathological examination begins with the preparation of highquality tissue sections. Several factors critically contribute to increase or decrease the quality of the final analysis, including fixation, macroscopic sectioning, processing, paraffin embedding, and embedding of the samples ${ }^{6,7}$.

All these passages involving manipulation of the sample are subjected to manual errors, including manual embedding of the samples and, to a lesser extent, manual microtome sectioning and staining. At present, the whole process of murine tissue preparation for histological evaluation relies on protocols that vary from laboratory to laboratory and manual protocols. The goal of this study is to implement standardized automated protocols to reduce errors and variability in murine histopathological examination.

To our knowledge, we describe here the first protocols for fully automated tissue processing and embedding for the histological evaluation of murine tissues; these are routinely used in pathology units for the analyses of human specimens. As a practical example of the feasibility of the method, a murine model of intestinal inflammation has been analyzed, i.e., the chronic colitis model caused by repeated administration of dextran sodium sulphate (DSS) in the drinking water ${ }^{8,9}$. This experimental setting closely resembles human inflammatory bowel diseases (IBD) ${ }^{10}$ since DSS-treated animals exhibit signs of intestinal inflammation, e.g., weight loss, loose stool or diarrhea, and shortening of the colon as well as fibrosis ${ }^{8,9,11}$. As observed for human IBD patients, DSS treatment generates a complex disease course. In this context, elaborate histological evaluations are required to understand the profound alteration of the tissue architecture. Thus, the implementation of the described protocols for 
increasing sample preparation quality might benefit researchers relying on the interpretation of histological and immunohistochemical analyses for murine experimental settings. Murine experimental models of human diseases involving alterations of the tissue architecture, the presence of cellular tissue infiltrate or inflammation in different tissues and organs (intestine, brain, liver, skin) could use the increased quality of the sample preparation for histopathological examination.

Protocol

Animal procedures were approved by the Italian Ministry of Health (Auth. 127/15, 27/13) and followed the animal care guidelines of the European Institute of Oncology IACUC (Institutional Animal Care and Use Committee)

\section{Chronic Colitis Induction by Repetitive DSS Administration}

1. Separate age and sex matched mice in 2 groups (treatment DSS vs. control $\mathrm{H}_{2} \mathrm{O}$, at least 5 mice littermates per experimental group).

2. Administer $2.5 \% \mathrm{DSS}(40 \mathrm{kDa})$ in the drinking water for 7 days to the treatment group and water to the control group. NOTE: This model induces a chronic transmural intestinal inflammation ${ }^{9}$.

3. After 7 days, stop DSS treatment and give water to both groups for 14 days. Repeat this process 3 times. NOTE: Repetitive administration of DSS induces alterations of the colonic mucosa closely resembling human IBD, i.e., fibrosis ${ }^{9}$

4. Sacrifice mice according to the procedures authorized by the Institutional IACUC, i.e., by $\mathrm{CO}_{2}$ inhalation.

5. Open the mouse abdomen and peritoneum with a scalpel. NOTE: Sterility is recommended but not strictly required.

6. Separate the colon from the small intestine with forceps and tweezers. Excise the colon with tweezers and forceps. NOTE: Colon length measurement (Figure 1A) is a method to determine if colitis occurred in DSS-treated mice.

7. Rinse the colon in a Petri dish with $10 \mathrm{~mL}$ of cold $1 \mathrm{x}$ phosphate buffer saline (PBS) and gently press to remove fecal material.

\section{Murine Tissues Fixation}

1. Immerse each murine colon specimen in $10 \mathrm{~mL}$ of $10 \%$ Neutral Buffered Formalin (NBF) at RT (room temperature). NOTE: Sterility is recommended but not strictly required.

2. Fix the tissue for $18-24 \mathrm{~h}$ at RT.

\section{Colon Sectioning and Tissue Preparation}

1. Remove fixed tissues from the NBF container with small tweezers and put them in a Petri dish. Put the colon on a sectioning work plate with small tweezers.

2. Cut colons in fragments $(0.2 \mathrm{~cm}$ to $0.3 \mathrm{~cm}$ lengths) with a sterile scalpel. This fragment length is optimal in order not to exceed the thickness of the cassette.

3. Pick up one colon segment with small tweezers (Figure 2A). Insert one colon segment into one of the plastic protruding tips of the orientated paraffin embedding cassette with small tweezers (Figure 2B).

4. Repeat the operation (3.2-3.3) with an additional 3 colon segments per cassette. Avoid inserting the segments into adjacent protruding tips, to minimize overlapping of the tissues

5. Tightly close the cassette by carefully pushing the four edges (Figure 2C). Avoid squeezing the tissue to prevent tissue damage.

6. Insert the grid into a plastic supporting frame. Label the supporting frame to identify the sample. Repeat for each biological sample.

\section{Tissue Processing}

1. Turn on the automated processor by pushing the power button (Figure 3A, 3B).

2. Warm up for $1 \mathrm{~h}$ to ensure paraffin wax melting. Wait until the instrument confirms the paraffin is completely melted, by observing the presence of the dedicated icon (Figure $3 \mathbf{C}$ ).

3. Insert each oriented cassette (containing the tissue specimens) manually into the metal basket provided by the automated processor (Figure 3D). Place the cassettes vertically by lining them close to one to the other to optimize the basket occupancy.

4. Close the metal basket (Figure 3E).

5. Open the lid of the retort (Figure $\mathbf{3 F}$ ). The retort is the place where the basket is inserted into the machine. Insert the basket into the dedicated housing of the processor (Figure $\mathbf{3 G}$ ). Close the lid of the retort (Figure $\mathbf{3 H}$ ).

6. Use the touch screen on the instrument computer to define the working protocol (Figure 3I). Choose the sequence of solutions, timing and temperature to be implemented according to the scheme provided in Table 1.

7. Assign the protocol to be run on the retort containing the basket by clicking on the dedicated computer icon (Figure $3 \mathbf{J}$ ). Start the protocol by clicking on the Start Button (Figure 3L).

8. Wait until the instrument confirms the end of the protocol, by observing the presence of the dedicated icon and by hearing the alarm tone coming from the machine.

9. Open the retort lid. Remove the basket from the processor (Figure 3M)

\section{Tissue Embedding}

1. Turn on the automated embedder by pushing the power button (Figure 4A).

2. Warm up for $1 \mathrm{~h}$ to ensure paraffin wax melting. Wait until the instrument confirms the paraffin is completely melted by observing the temperature of the paraffin bath indicated by the internal thermometer of the instrument. 
3. Manually transfer all the processed cassettes from the processor basket to the embedder rack. Each rack can contain up to 32 cassettes. (Figure 4B, 4C).

4. Open the main embedder lid (Figure 4C, 4D, 4E)

5. Use the touch screen on the instrument computer to signal to the robotic system that a rack is being inserted (Figure $\mathbf{4 F}$ ).

6. Open the inlet housing lid (Figure $\mathbf{4 G}$ ). Insert the rack into the inlet housing. Each embedder can contain up to 4 racks simultaneously (Figure 4H, 4I). Close the inlet housing lid (Figure 4J).

7. Use the touch screen on the instrument computer to start the embedding procedure, according to Table 2 (Figure 4K). NOTE: Each rack of 32 cassettes takes 45 min to be embedded.

8. Wait until the instrument confirms the end of the protocol, by observing the presence of the dedicated icon (Figure 4L).

9. Remove the outlet rack (Figure 4M). Close the main embedder lid.

10. Remove the embedded blocks (containing the orientation grids) from the rack. Transfer the embedded blocks in a storage cardboard box.

\section{Micrometer Sectioning}

1. Turn on the cooling plate of the microtome. Set the temperature between -8 and $-10{ }^{\circ} \mathrm{C}$.

2. Turn on the thermostatic water bath of the microtome, containing $2 \mathrm{~L}$ of distilled water. Set the temperature of the water bath between $42-45$ ${ }^{\circ} \mathrm{C}$

3. Place the paraffin embedded blocks on the cooling plate. Wait at least $5 \mathrm{~min}$ to allow the blocks to cool.

4. Take one block from the cooling plate and place it into the microtome block holder

5. Set the microtome cut thickness to $10 \mu \mathrm{m}$. Trim the block by cutting it 6 times at $10 \mu \mathrm{m}$ thickness.

6. Change the thickness setting of the microtome from $10 \mu \mathrm{m}$ to $3 \mu \mathrm{m}$. Cut one $3 \mu \mathrm{m}$ section for Hematoxylin and Eosin (H\&E) staining.

7. Collect the $3 \mu \mathrm{m}$ section with a small brush. Put the $3 \mu \mathrm{m}$ section in the water bath, laying it carefully on the water surface to reduce tissue wrinkles. Collect the tissue section from the thermostatically controlled water bath on a single glass slide.

1. If needed, cut additional sections.

8. Label the glass slide by writing the sample identification.

9. Put the slides in a $37^{\circ} \mathrm{C}$ oven for at least $10 \mathrm{~min}$.

10. Put glass slides into racks for immediate analyses or in storage boxes.

\section{Hematoxylin and Eosin (H\&E) Staining}

1. Switch on the automated stainer by pushing the power button. Allow the initialization of the robotic arm, by observing the change of the loading bar on the instrument monitor.

2. Insert the glass slides into the stainer rack, vertically inserting up to 30 slides

3. Label the rack with the appropriate radio frequency identification (RFID) plastic tag. RFID tagging is a system identifying the correct staining protocol loaded into the stainer computer.

4. Insert the rack into the stainer. Allow the computer to automatically load and start the staining protocol according to the recognition of the RFID tag.

NOTE: The protocol for H\&E staining is described in Table 3.

\section{Immunohistochemical Staining}

1. Perform immunohistochemical and Mallory trichrome staining as previously described ${ }^{7}$.

\section{Representative Results}

Experimental chronic colitis induced by repeated administration of DSS in the drinking water is a murine model of intestinal inflammation closely resembling human $\mathrm{IBD}^{8,9}$. Figure 1 describes the effects of DSS treatment, including colon shortening (Figure 1A), a widely-used parameter to score the presence of DSS-induced inflammation, and colonic expression of pro-inflammatory genes including $C X C L 10, t n f$ and $m c p-1$ (Figure 1B). Infiltration of inflammatory cells was greatly enhanced by DSS treatment, showing a recruitment of the immune response in the intestinal lamina propria as analyzed by cytofluorimetry (Figure 1C).

Figure 2 depicts how colonic tissues are sectioned and inserted in the oriented cassettes. These cassettes are designed to contain a grid with extruded tips, allowing the insertion of the tissue vertically and with the proper orientation, i.e., with the lumen in the inner part and the wall exteriorly (Figure 2A, 2B, 2C). Once the cassettes are closed, the tissue orientation is preserved by the grids, contrary to what happens with conventional histological cassettes (Figure 2B).

The tissue processing is performed with an automated processor (Figure 3A-3M and Table 1), while the embedding procedure is performed with an automated embedder (Figure 4A-4M and Table 2). In the latter instrument, a robot collects the cassettes and dispenses the correct amount of paraffin. Finally, by using a microtome, sections are cut from each paraffin block. The slides are then prepared for further analysis. 
Figure 5 describes the main passages of the automated H\&E staining (Figure 5A-5E and Table 3 ) and how the slides appear after H\&E staining (Figure 5F). Figure 6 and 7 show how the implementation of the automated processing and embedding protocols strongly increase the quality of the histopathological analyses of murine colonic specimens. The H\&E stainings of samples prepared with the automated protocols derived from untreated mice (Figure 6A) were compared to those of DSS treated mice (Figure 6B). Histopathological scores were assessed by evaluating the modifications of different parameters occurring in the intestinal mucosa, including inflammatory cells infiltration, epithelial alterations and changes of the mucosal architecture as described in Table 4. Figure 6C depicts a representative H\&E staining of a colonic tissue processed manually and included in traditional cassettes. In Figure 7, the quality of the murine tissue preparation and embedding performed through automated instruments was additionally confirmed by IHC staining of CD20 and Mallory trichrome staining ${ }^{8,9}$ in colonic sections of untreated (Figure 7A, 7C) and DSS-treated (Figure 7B, 7D) mice.

Figure 8 describes the practical relevance of this method. The same colon samples were processed and embedded either manually or through automatic methods. Each sample (either the control or DSS-treated) was cut into 2 equal parts and processed in parallel with the manual or with the automatic methods. H\&E staining was then performed and a complete microscopical evaluation concerning all the pathological parameters addressing changes in the mucosal architecture, granularity, immune cell infiltrate, mucosal thickness, glandular rarefaction normally observed during intestinal inflammation, was assessed for each sample, both for the manual and automated protocol (Figure 8A and Table 4). The preparation of the samples with the automated protocol consistently allowed the evaluation of a higher proportion of histological parameters than the manual method. Additionally, a separate analysis of different histological parameters was performed (Figure 8B). The architecture and the basal infiltrate evaluation were positively affected, especially in untreated mice, by the sample preparation with the automated method.

The automated method for sample processing and embedding currently used for human histopathological analyses can be successfully applied for the analysis of murine specimens. High quality specimens are generated with the automated method that demonstrates the superiority over the manual method in the assessment of the architecture and the basal immune infiltrate of murine colonic tissues.

A

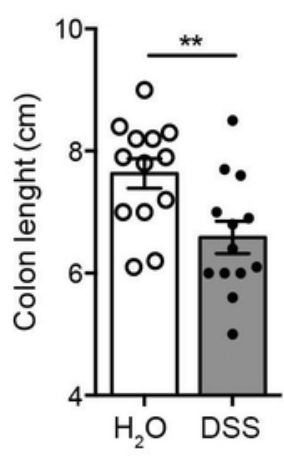

C

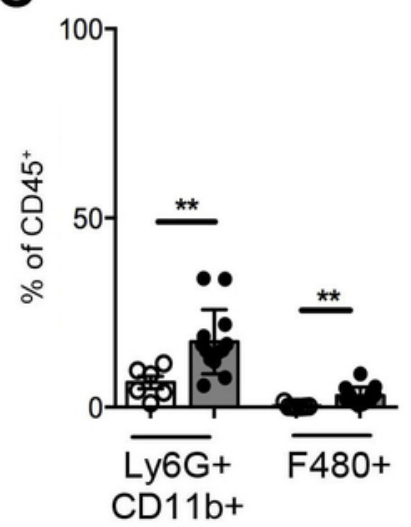

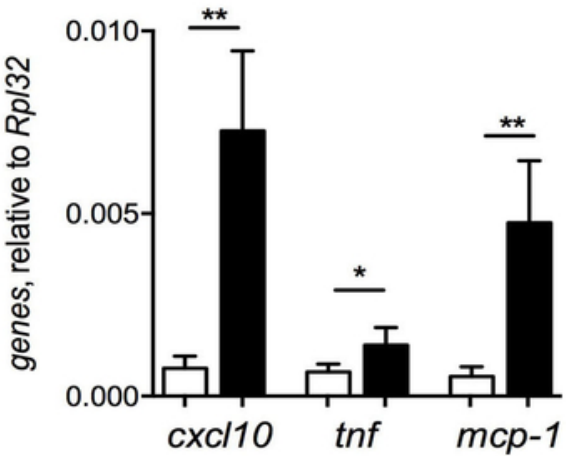

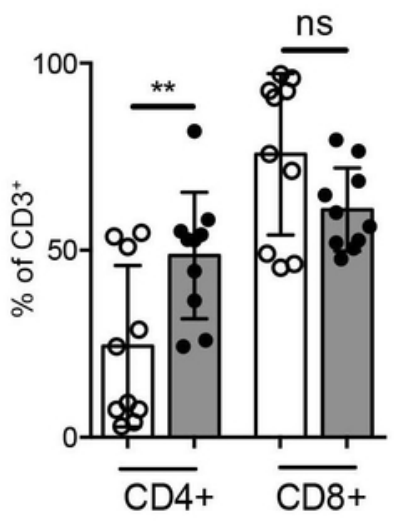

$\square \mathrm{H}_{2} \mathrm{O}$

DSS

Figure 1: Chronic intestinal inflammation evaluation. (A) Colon length measurement. (B) Colonic expression of pro-inflammatory genes (cxcl10, tnf, $m c p-1$ ) in DSS-treated (black bars, $n=12$ ) and untreated mice (white bars, $n=6$ ). (C) Immunophenotyping of colonic lamina propria cells in DSS-treated (closed symbols, $n=12$ ) and untreated mice (open symbols, $n=6$ ). CD11b+Ly6G+ neutrophils, CD11b+F4/80+ macrophages (left panel), CD4+ and CD8+T cells (right panel) infiltration in controls (open symbols, $n=10$ ) and DSS-treated mice (closed symbols, $n=10)$. Statistical significance was calculated using Wilcoxon matched-pairs signed rank t test. ${ }^{*} p \leq 0.05{ }^{* *} p \leq 0.01$. Mean value \pm SEM are reported. Please click here to view a larger version of this figure. 

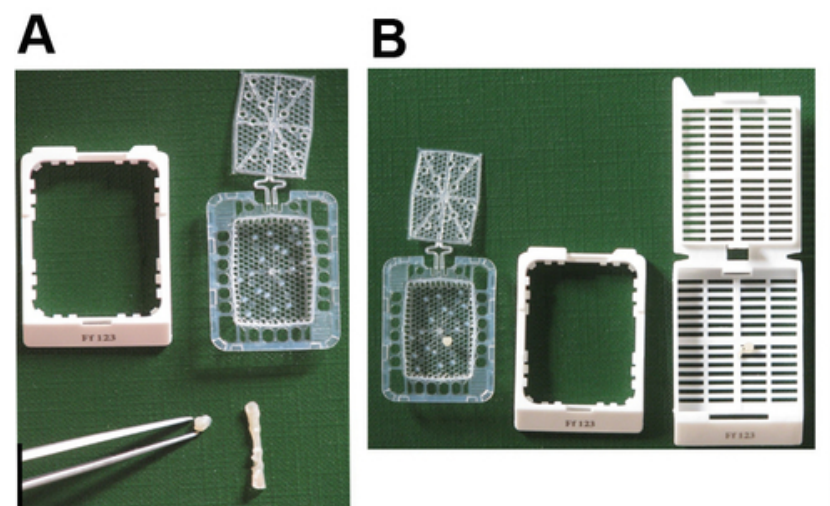

C

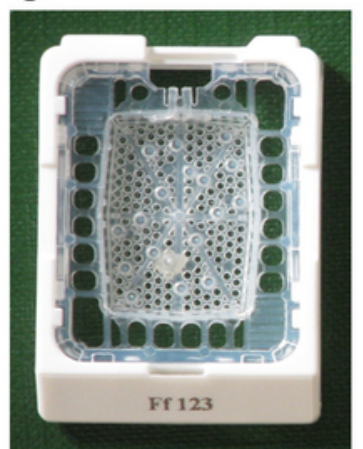

Figure 2: Description of sample preparation. (A) Instruments required for tissue preparation and picture of the orientated cassette, composed by an external cassette (white) and internal grid with extruded orientation tips. (B, C) Insertion of murine colons in the internal grids of the cassette before (B) and after (C) closure of the grids. In panel B is depicted the orientated cassette (left) or in a traditional cassette (right). Please click here to view a larger version of this figure. 
A

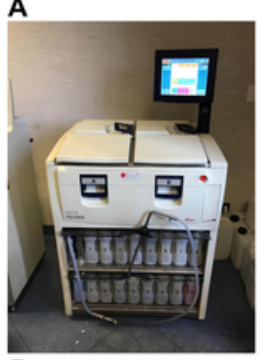

D

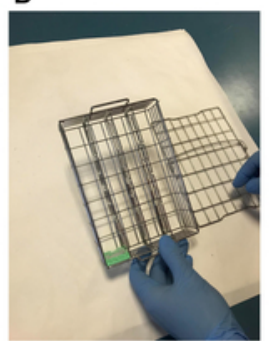

H

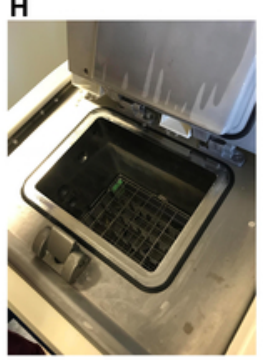

L

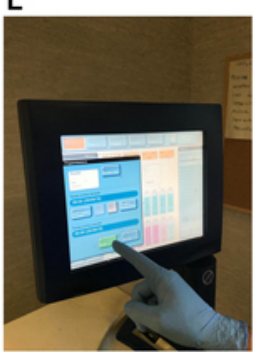

B

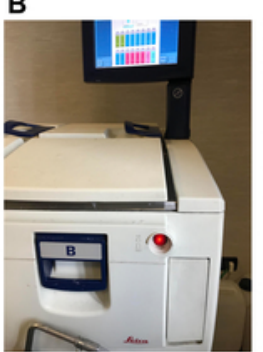

E
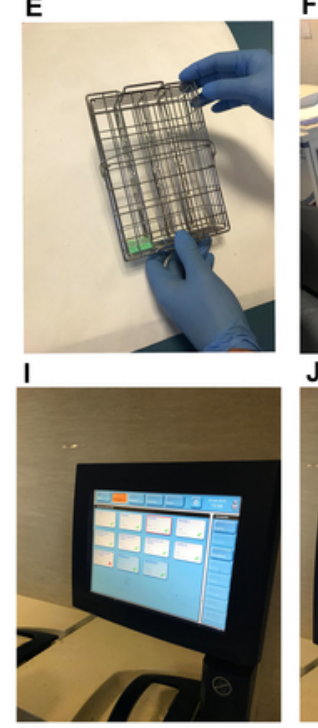

M

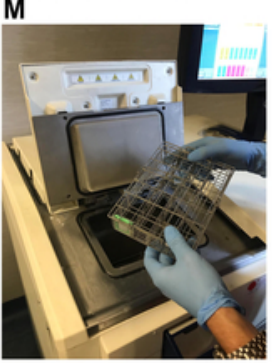

C

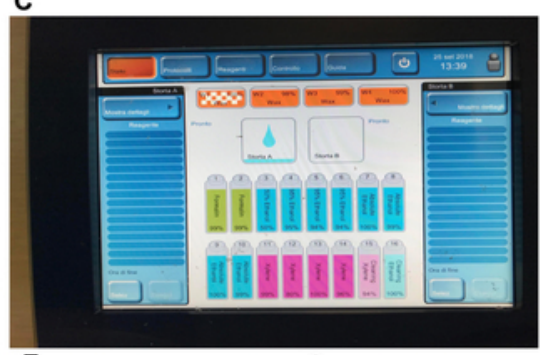

F

G

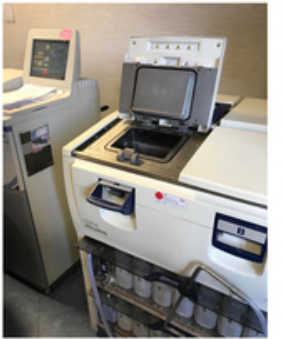

$\mathrm{J}$

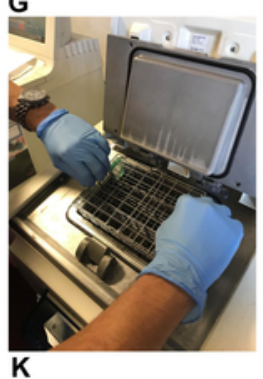

K
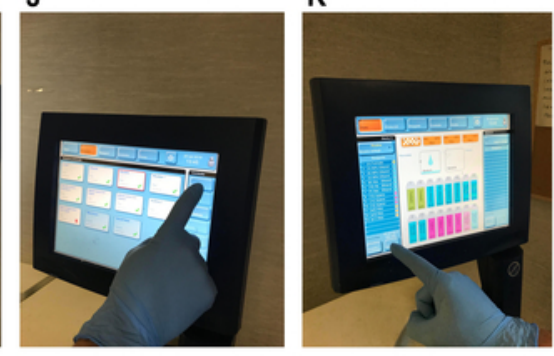

Figure 3: Description of automated processing. (A, B) automated processor. (C) Icon describing the correct paraffin wax melting temperature. (D, E) Insertion of the cassette in the metal basket (D) and its closure (E). (F, G, H) Insertion of the metal basket in the retort. (I, J, K) Selection of the processing protocol. (L) Running of the protocol. (M) Removal of the basket form the retort. Please click here to view a larger version of this figure. 
A

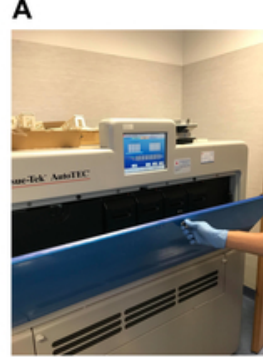

$\mathrm{E}$

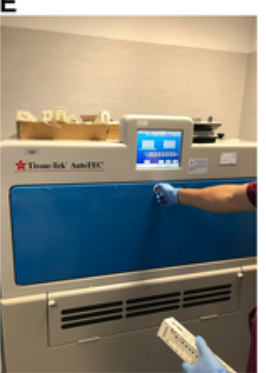

I

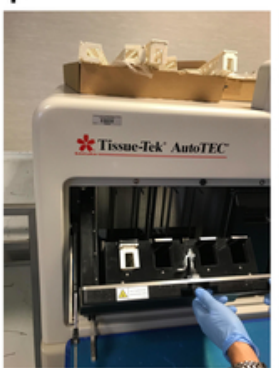

M
B
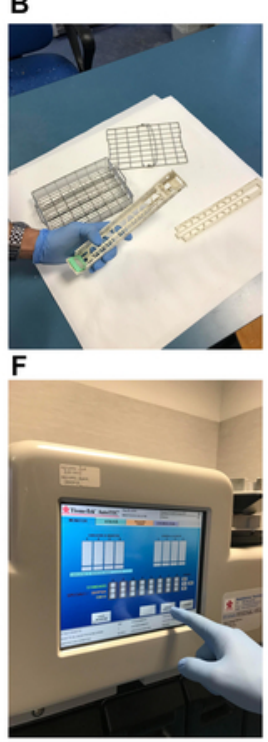

J

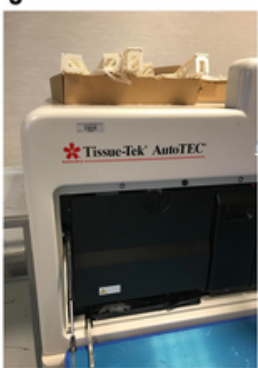

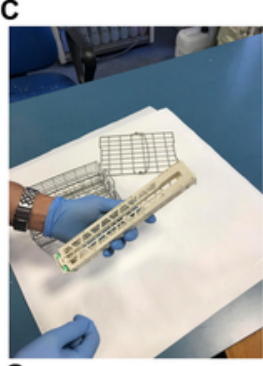

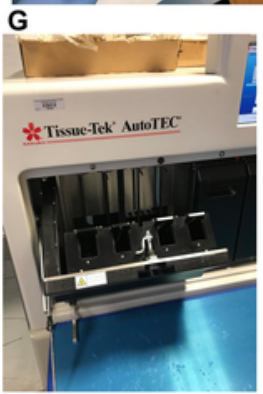

K

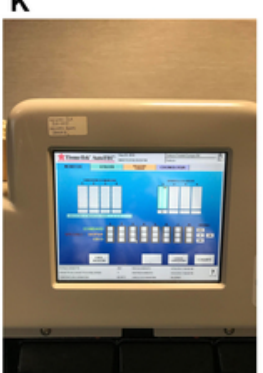

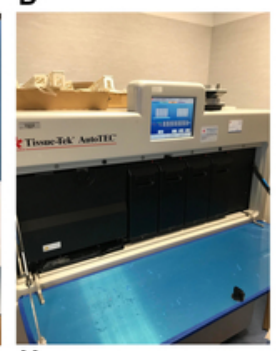

H
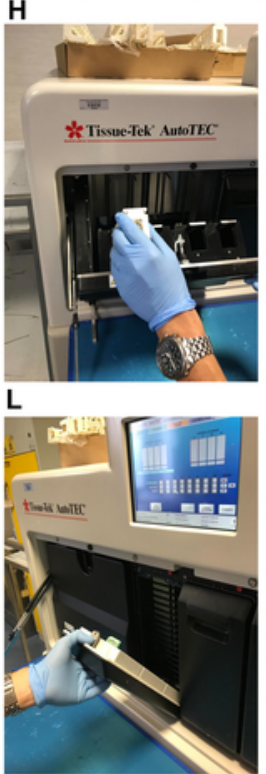

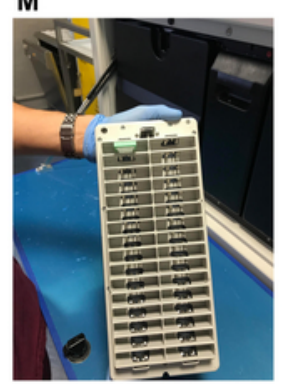

Figure 4: Description of automated embedding. (A) Picture of the automated embedder. (B, C) Insertion of the cassettes in the rack. (D, E) Opening (D) and closing (E) of the lid. (F) Signaling to the machine of the presence of a rack. (G, $\mathbf{H})$ Insertion of the rack into the inlet housing. (I, J) closing of the lid. (K) Start of the embedding protocol. (L) Opening of the outlet housing lid. (M) Removal of the rack form the outlet housing. Please click here to view a larger version of this figure. 
A

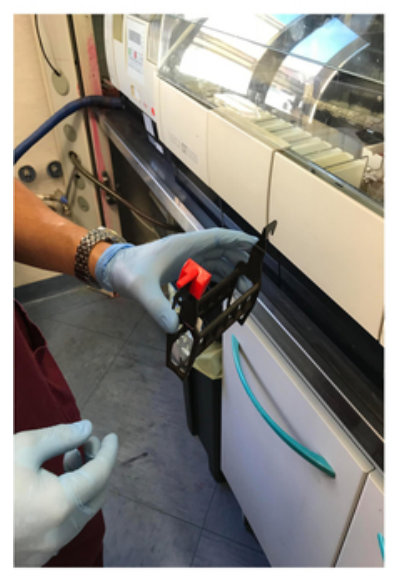

D

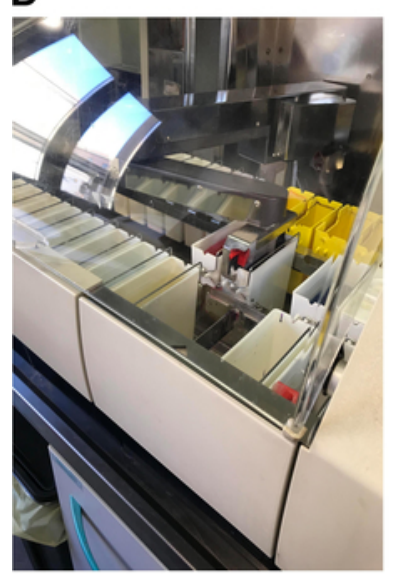

B

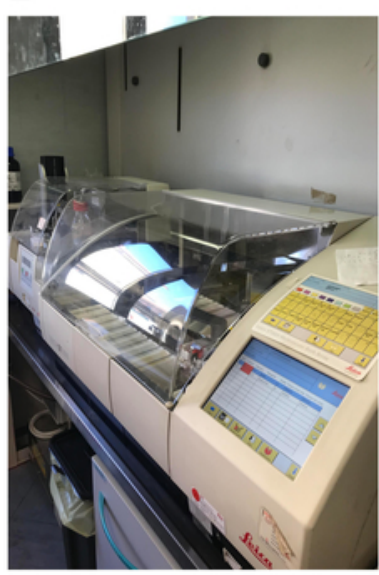

E

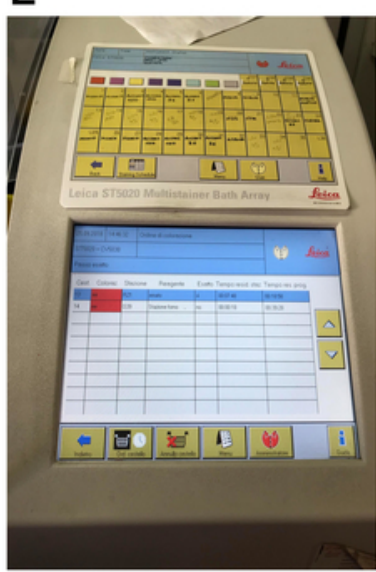

C
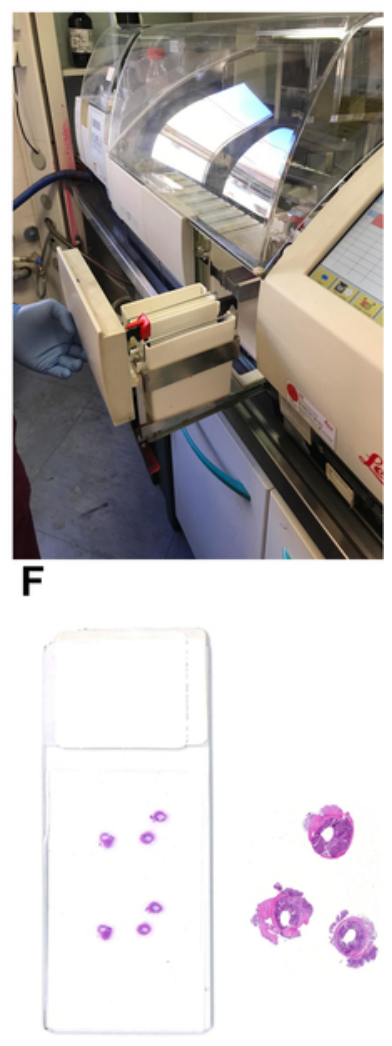

Figure 5: Description of automated H\&E stainer. (A) Picture of the slide holder. (B, C) Insertion of the slide holder in the machine. (D) Closing of the machine. (E) Start of the staining protocol. (F) Exemplificative picture of a slide after microtome cutting and H\&E staining. Right panel, H\&E staining depicting the sample orientation. Please click here to view a larger version of this figure. 
A

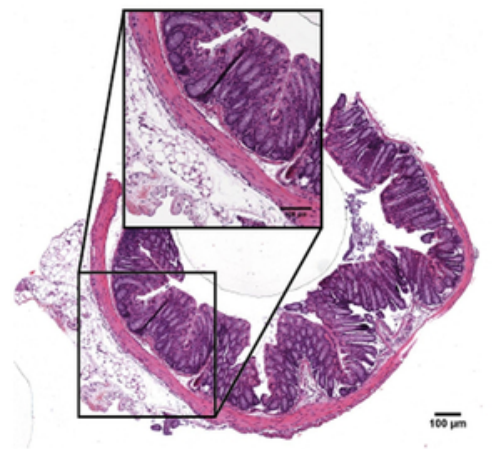

B

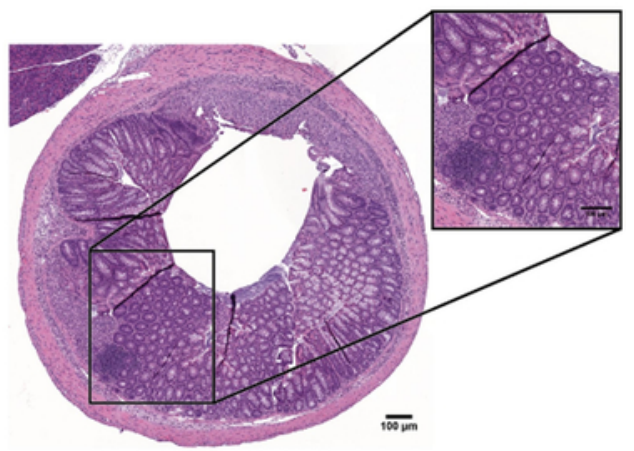

C

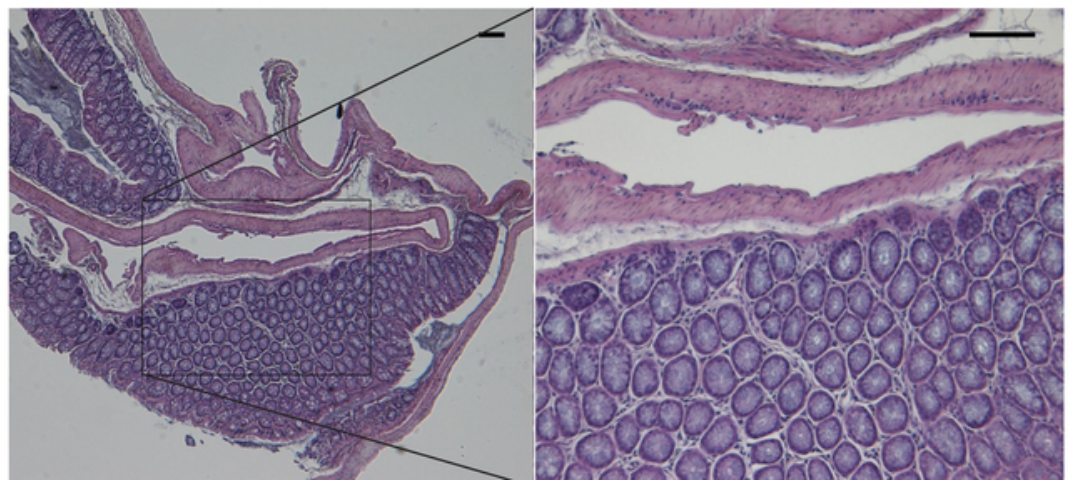

Figure 6: H\&E stainings of colonic samples form untreated and DSS-treated mice. H\&E staining of untreated (A) and DSS-treated (B) samples prepared (processed, embedded, stained) with automated (A, B) or manual (C) methods. Scale bar $=100 \mathrm{~nm}$. Please click here to view a larger version of this figure.

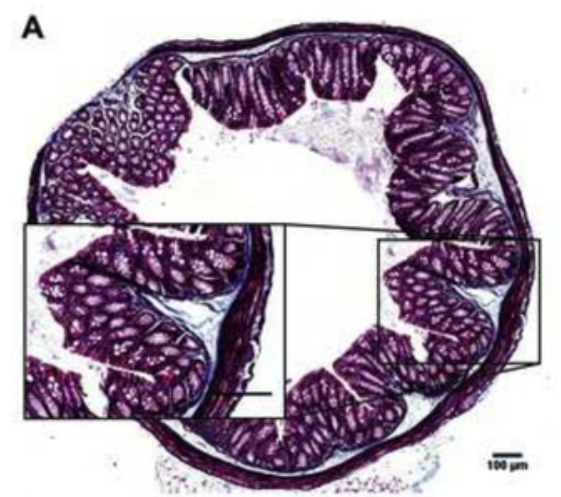

C

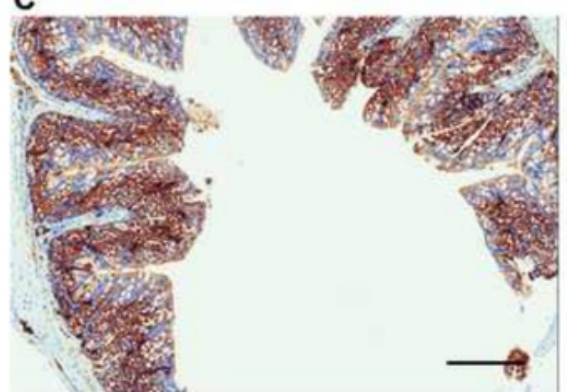

B

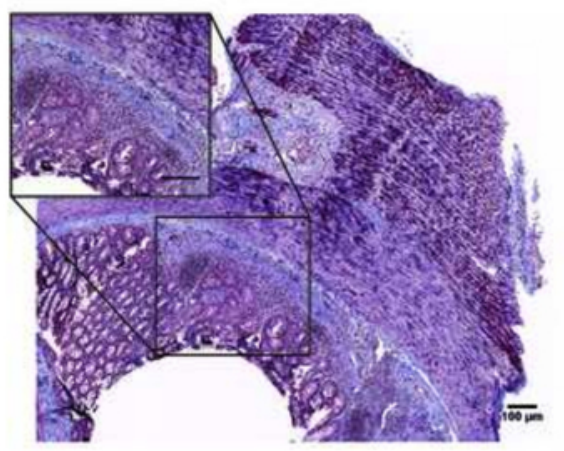

D

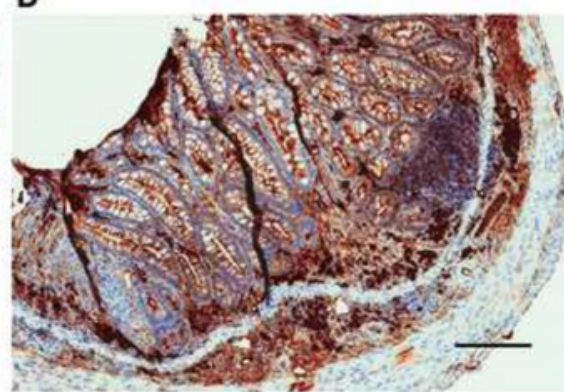

Figure 7: Mallory trichrome (A, B) and IHC stainings of infiltrating CD20+ cells (C, D) of untreated (A-C) and DSS treated (B, D) mice. Mallory staining: Blue, collagen, Dark pink, nuclei, Dark red, cytoplasm. Scale bar $=100 \mathrm{~nm}$. Please click here to view a larger version of this figure. 
A

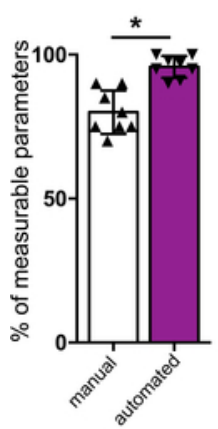

B

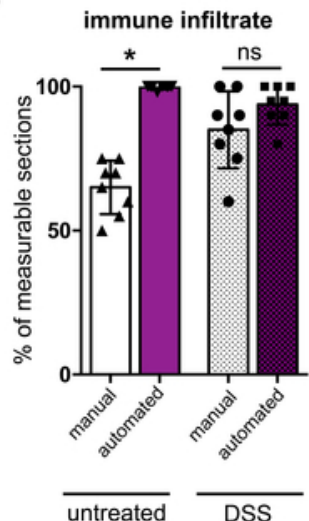

mucosal thickness
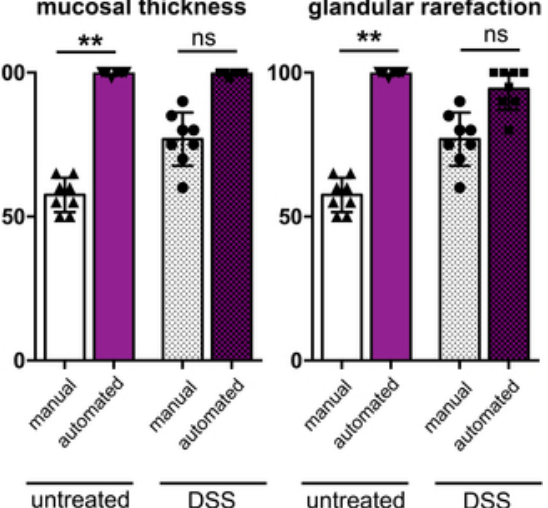

Figure 8: Comparison between histopathologic analyses with manual and automated protocols. (A) Total histopathological parameters assessable in all the sections prepared either with the manual (white bars) or with the automated method (purple bars). (B) Percentage of the indicated parameters in the samples prepared with the manual (white bars) or with the automated method (purple bars) in untreated (Plain bars) or DSS-treated mice (dotted bars). Statistical significance was calculated using Wilcoxon matched-pairs signed rank t test. * $p<0.05 ;{ }^{* *} p<$ 0.005 . Mean value \pm SEM are reported. Please click here to view a larger version of this figure.

\begin{tabular}{|l|l|l|l|}
\hline Reagent & time (min) & temperature $\left.{ }^{\circ} \mathbf{C}\right)$ & pressure \\
\hline NBF & 1 & RT & ambient \\
\hline Ethanol 95\% & 1 & RT & ambient \\
\hline Ethanol 95\% & 1 & RT & ambient \\
\hline Ethanol 95\% & 1 & RT & ambient \\
\hline Absolute Ethanol & 1 & 45 & ambient \\
\hline Absolute Ethanol & 11 & 45 & ambient \\
\hline Absolute Ethanol & 30 & RT & ambient \\
\hline Xylene & 1 & RT & ambient \\
\hline Xylene & 1 & 45 & ambient \\
\hline Xylene & 28 & 45 & ambient \\
\hline Paraffin wax & 5 & 65 & vacuum \\
\hline
\end{tabular}

Table 1: Automated tissue processing protocol.

\begin{tabular}{|l|}
\hline Action perfomed by the robot for each cassette \\
\hline Remove one cassette from the rack \\
\hline Identify the cassette \\
\hline Pre-heat the mold \\
\hline Place the cassette on the pre-heated mold \\
\hline Dispense the amount of paraffin for the cassette \\
\hline Cool down the mold \\
\hline Allow the paraffin to solidify \\
\hline Remove the solidified paraffin block from the mold \\
\hline Present the block to quality sensors \\
\hline Place the paraffin block in the output door \\
\hline
\end{tabular}

Table 2: Automated embedding protocol. 


\begin{tabular}{|c|c|c|c|}
\hline Category & Criterion & Definition & Score value \\
\hline \multirow[t]{7}{*}{ Inflammatory cell infiltrate } & $\begin{array}{l}\text { Severity (leukocyte density of } \\
\text { lamina propria area infiltrated in } \\
\text { evaluated } \mathrm{hpf} \text { ) }\end{array}$ & No infiltrate & 0 \\
\hline & & Minimal acute $(<10 \%)$ & 0.25 \\
\hline & & $\begin{array}{l}\text { Mild chronic (10-25\%, scattered } \\
\text { neutrophils) }\end{array}$ & 0.5 \\
\hline & & Moderate chronic (26-50\%) & 0.75 \\
\hline & & Marked (>51\%, dense infiltrate) & 1 \\
\hline & $\begin{array}{l}\text { Extent (expansion of leukocyte } \\
\text { infiltration) }\end{array}$ & Mucosal & 0.5 \\
\hline & & Mucosal and submucosal & 0.75 \\
\hline \multirow[t]{10}{*}{ Epithelial changes } & $\begin{array}{l}\text { Hyperplasia (increase in epithelial } \\
\text { cell numbers in longitudinal crypts, } \\
\text { visible as crypt elongation) }\end{array}$ & No hyperplasia & 0 \\
\hline & & Minimal $(<25 \%)$ & 0.25 \\
\hline & & Mild (26-35\%) & 0.5 \\
\hline & & $\begin{array}{l}\text { Moderate }(36-50 \%, \text { mitoses in the } \\
\text { upper third of the crypt epithelium) }\end{array}$ & 0.75 \\
\hline & & $\begin{array}{l}\text { Marked ( }>51 \%, \text { mitoses in crypt } \\
\text { epithelium distant from crypt base) }\end{array}$ & 1 \\
\hline & $\begin{array}{l}\text { Goblet cell loss (reduction of goblet } \\
\text { cell numbers relative to baseline } \\
\text { goblet cell numbers per crypt) }\end{array}$ & No loss & 0 \\
\hline & & Minimal $(<25 \%)$ & 0.25 \\
\hline & & Mild (26-35\%) & 0.5 \\
\hline & & Moderate (36-50\%) & 0.75 \\
\hline & & Marked $(>51 \%)$ & 1 \\
\hline \multirow[t]{11}{*}{ Mucosal architecture } & $\begin{array}{l}\text { Ulceration (epithelial defect } \\
\text { reaching beyond muscolaris } \\
\text { mucosae) }\end{array}$ & No ulcers & 0 \\
\hline & & Ulcers & 0.25 \\
\hline & $\begin{array}{l}\text { Granulation tissue (connective } \\
\text { tissue repair with new capillaries, } \\
\text { surrounded by infiltrating cells, } \\
\text { hypertrophied areas) }\end{array}$ & No granulation tissue & 0 \\
\hline & & Granulation tissue & 0.25 \\
\hline & Mucosal thickness and crypt depth & No thickening & 0 \\
\hline & & Thickening & 0.5 \\
\hline & Glandular rarefaction & No rarefaction & 0 \\
\hline & & Rarefaction & 0.5 \\
\hline & Dysplasia & No dysplasia & 0 \\
\hline & & Dysplasia & 0.5 \\
\hline & & MAX SCORE & 6 \\
\hline
\end{tabular}

Table 3: Automated staining protocol. 


\begin{tabular}{|c|c|c|c|}
\hline $\begin{array}{l}\text { Action perfomed by the stainer } \\
\text { for each slide }\end{array}$ & Reagent & time (s) & temperature $\left({ }^{\circ} \mathrm{C}\right)$ \\
\hline Essicate & & 180 & 60 \\
\hline Essicate & & 180 & 60 \\
\hline Deparaffinize & Xylene & 120 & RT \\
\hline Deparaffinize & Xylene & 120 & RT \\
\hline Hydrate & Ethanol 96\% & 120 & RT \\
\hline Hydrate & Ethanol 96\% & 120 & RT \\
\hline Wash & Distilled wtaer & 240 & RT \\
\hline Stain Hematoxylin & Carazzi's Hematoxylin & 540 & RT \\
\hline Rinse & Tap Water & 360 & RT \\
\hline Stain Eosin & Eosin $\mathrm{Y} 1 \%$ aqueos solution & 60 & RT \\
\hline Rinse & Tap Water & 120 & RT \\
\hline Dehydrate & Ethanol 96\% & 20 & RT \\
\hline Dehydrate & Ethanol 96\% & 20 & RT \\
\hline Dehydrate & Absolute Ethanol & 15 & RT \\
\hline Dehydrate & Absolute Ethanol & 15 & RT \\
\hline Clear & Xylene & 30 & RT \\
\hline Clear & Xylene & 30 & RT \\
\hline
\end{tabular}

Table 4: Scoring scheme for the evaluation of intestinal inflammation.

\section{Discussion}

We utilize different automated steps during the preparation of murine tissues for histopathologic analysis. This protocol aims at providing technical hints to increase the reproducibility and the standardization of the whole process, thus enhancing the overall quality of the final histopathological evaluation. We implemented automated instruments and methods for the preparation and embedding of tissues, routinely used in pathology core facilities for the study of human specimens.

To demonstrate the potential applicability of this method, we chose a chronic murine experimental setting of intestinal inflammation, called DSSinduced chronic colitis model. This setting resembles the complex disease course observed in IBD patients, requiring elaborate histological evaluation to investigate the profound alterations of the tissue architecture occurring upon chronic intestinal inflammation. Altogether, the histological evaluation of these processes might greatly benefit from increased sample preparation quality. To note, this protocol can be applied to other murine tissues (i.e., spleens, lymph nodes, liver, brain), with the only difference being that the oriented cassette is not needed for tissues without a lumen.

The most important observation was that the decrease of manual errors, (i.e., the orientation of the sample) given the use of cassettes with grids and the elimination of cassette re-opening for the embedding (a step commonly performed during manual protocols), strongly enhanced the overall reproducibility of the analysis. With the protocol described here, the sample is manipulated only at the beginning of the preparation, when the experimenter inserts the tissue in the orientated cassettes. Once closed, the cassettes are never re-opened, thus ensuring the maintenance of the correct orientation and reducing manual errors ${ }^{3,11}$. The standardization of these two critical steps enhanced the whole quality of the subsequent analyses and decreased the number of lost or not assessable samples, problems both linked to the re-opening of the cassette or to the wrong orientation of the sample ${ }^{3,11}$.

We also succeeded in evaluating a complex biological event such as fibrosis (Figure 7), that is very often underestimated in murine models of experimental intestinal inflammation, by the implementation of the automated protocol. During the set-up of the protocol, we strictly standardized technical details such as the fixation time, which we realized must not exceed $24 \mathrm{~h}$ to avoid tissue alterations. By doing so, we could preserve the quality of subsequent immunohistochemical analyses.

The automated method improved the evaluation of those parameters associated to the alteration of the tissue architecture, especially in untreated mice. Indeed, the correct comparison of a pathological tissue with a healthy counterpart is critically important for the final assessment of the experimental model ${ }^{3}$.

Concerning the tissue processing, we tested different protocols to be run in the automated processor available in the laboratory. The protocol described here gave sound and highly consistent results. We also implemented a time length for the protocol. Since murine samples were comparable to human small biopsic specimens in size, a shorter protocol was sufficient to process murine (intestinal, in this case) tissues. Finally, the whole automated procedure reduced the total experimental time. From the beginning of the processing to the microtome cutting and slices preparation, we calculated that the total time required is $5 \mathrm{~h}$. On the contrary, depending on the technical ability of the operator, the completion of the same protocols manually can require more than double the amount of time, especially during the processing and the embedding. One limit of the technique is that it requires automated processors and embedder to be performed to be in the laboratory. 
In conclusion, we believe that the implementation of these automated protocols could greatly ameliorate the work of translational researchers dealing with murine experimental models of human diseases.

\section{Disclosures}

The authors have nothing to disclose.

\section{Acknowledgements}

We thank the department of Pathology of the IRCCS Policlinico Hospital, Milan for technical support and the IEO Animal Facility for assistance in animal husbandry.

\section{References}

1. Gibson-Corley, K.N. et al. Successful Integration of the Histology Core Laboratory in Translational Research. Journal of histotechnology. 35, 17-21 (2012).

2. Olivier, A.K. et al. Genetically modified species in research: Opportunities and challenges for the histology core laboratory. Journal of histotechnology. 35, 63-67 (2012).

3. Gibson-Corley, K.N., Olivier, A.K., \& Meyerholz, D.K. Principles for valid histopathologic scoring in research. Veterinary pathology. 50, 1007-1015 (2013).

4. Stolfi, C. et al. Involvement of interleukin-21 in the regulation of colitis-associated colon cancer. The Journal of experimental medicine. 208, 2279-2290 (2011).

5. Begley, C.G., \& Ellis, L.M. Drug development: Raise standards for preclinical cancer research. Nature. 483, 531-533 (2012).

6. Peters, S.R. A Practical Guide to Frozen Section Technique. In: New York, N.S.N.Y. (ed). (2010).

7. Rosai, J. Rosai and Ackerman's Surgical Pathology. Elsevier (2011).

8. Blumberg, R.S., Saubermann, L.J., \& Strober, W. Animal models of mucosal inflammation and their relation to human inflammatory bowel disease. Current opinion in immunology. 11, 648-656 (1999).

9. Wirtz, S., Neufert, C., Weigmann, B., \& Neurath, M.F. Chemically induced mouse models of intestinal inflammation. Nature protocols. 2, 541-546 (2007).

10. Kaser, A., Zeissig, S., \& Blumberg, R.S. Inflammatory bowel disease. Annual review of immunology. 28, 573-621 (2010).

11. Cribiù FM, B.C., et al. Implementation of an automated inclusion system for the histological analysis of murine tissue samples: A feasibility study in DSS-induced chronic colitis. European Journal of Inflammation. 16, 1-12 (2018). 